\title{
Chronic Inflammation: An Issue to Consider For Clinical Imaging In Cancer Care
}

\author{
*Mikhail Liubchak \\ Department of Radiology, Odrex Private Medical Center, Ukraine
}

Submission: March 24, 2017; Published: March 30, 2017

"Correspondence Address: Mikhail Liubchak, Radiologist, Odrex Private Medical Center, Odessa, Ukraine, Email: m.lyubchak@gmail.com

\begin{abstract}
This paper is an attempt to think about introducing changes to the current clinical cancer imaging frameworks by shifting the scope from screening macroscopic cancer to screening the pre-cancer chronic inflammatory states. A very brief overview of chronic inflammation concepts, cancer imaging approaches and some modern techniques for inflammation imaging is provided.

Keywords: Cancer; Biomarkers; Inflammation; Chronic Inflammation; Medical Imaging; Molecular Imaging; Hybrid Imaging

Abbreviations: DNA: Deoxyribonucleic Acid; CT: Computed Tomography; PET: Positron Emission Tomography; MR: Magnetic Resonance; FDG: Fluordeoxyglucose
\end{abstract}

\section{Introduction}

Biomedical imaging is one of the key aspects of comprehensive cancer care, providing morphological, structural, metabolic and functional information [1]. The current role of clinical imaging in cancer care is mostly based on screening, staging, treatment monitoring and follow-up [1]. The whole process is aimed at identifying a lesion and characterizing it's structure, vascularity (contrast enhancement), some extent of metabolic activity (using 18F-FDG or similar agents) and signs of disease spread. The screening step in this sense represents a point of "first contact" with diagnostic imaging modalities. However, as the screening search is focused on identifying a potentially malignant lesion, it is always a "little late", since the key assumption of such an approach is that the lesion might already be present. A logical upgrade of the screening framework would be a shift from the assumption that the lesion is already present to an assumption that a lesion might develop in a certain patient, and potential site and pre-cancer state should be identified. This means that diagnostic imaging could try to shift its focus to the conditions that precede and influence cancer development, thus moving from structural to functional molecular imaging. Among the states that precede or foster cancer development, chronic inflammation should be chosen as a priority target. Chronic inflammation is known to act as an ancestor, promoter or a satellite of a great number of serious diseases and pathological conditions, including COPD, multiple sclerosis, Alzheimer's disease, cardiovascular disease, diabetes, cancer and many others [2-5].
Naturally, inflammation in its acute phase acts as a key defense mechanism against invasive pathogens, eliminating them and providing tissue repair [6]. The cascade of inflammation starts with release of pro-inflammatory cytokins, chemokines and leukotrienes by resident inflammatory and endothelial cells and is followed by increase in vascular permeability with subsequent macrophage and neutrophil infiltration [6,7]. The process is normally finalized by release of pro-resolving mediators, leading to apoptosis of inflammatory cells and termination of inflammatory response [6]. The process of inflammation itself is usually quite thoroughly regulated, and involves both the signals that start and promote the inflammation, as well as the signals that stop it [7]. The imbalance between these two types of signals results in uncontrolled inflammatory process [7]. In other words failure to resolve the inflammation turns it into the chronic phase, in which the cellular response is dominated by lymphocites, plasma cells and macrophages with varying morphology [8]. The latter, if activated persistently, may lead to tissue and DNA damage by producing growth factors, cytokines, reactive oxygen and nitrogen species [8]. Such a microenvironment with cell proliferation under the circumstances of continuous tissue/ DNA damage is predisposed for development of cancer through "metaplasia $\rightarrow$ dysplasia $\rightarrow$ cancer" pathway [8-11].

Potential algorithm, methods and imaging targets in chronic inflammation, current progress and limitations

Imaging of chronic inflammation could be applied at all the stages of cancer development process, starting from screening 


\section{Cancer Therapy \& Oncology International Journal}

potentially pre-cancer state and moving towards advanced disease, where chronic peritumoral inflammation takes a significant part in cancer spread [5].

For example, in case of pre-cancer state screening, a patient at risk should be identified considering his genetic predispositions, clinical and laboratory information. The macroscopic signs of cancer should be excluded with conventional low-dose or nodose screening methods. After that a specialized imaging might be applied to further investigate the patient. The specialized imaging workflow might encompass several steps, starting with basic identification of chronic inflammation sites and proceeding to evaluation of signs of dysplasia/early cancer. A set of biomarkers could be tested using several modalities for demonstration of functional state of the cells within the area of interest, presence of cell atypia e.t.c. The resulting data could be post processed and analyzed in order to assign a risk category for the inflammation site and plan the according therapeutic intervention.

In case of advanced cancer, sites of chronic peritumoral inflammation could be included in comprehensive tumor assessment, with an emphasis on pro-metastatic conditions, like presence of tumor-associated macrophages, which is known to positively correlate with reduced survival in most cancers [5], signs of epithelial-mesenchymal transition, vascularization, cell proliferation and migration, expression of characteristic receptors and chemical signals, e.t.c. The data could be utilized for therapy planning/ therapy response evaluation, as well as for prognostic purposes and expansion of traditional tumorassociated classifications and prognostic indicators.

The imaging modalities to be used for visualization of chronic inflammation should likely by hybrid (like PET-CT or PET-MR), utilizing both highest metabolic sensitivity and highest possible spatial resolution. PET and nuclear medicine techniques currently are the most sensitive with between nanomole/kilogram and picomole/kilogram sensitivity, while CT and MRI systems that provide highest spatial resolution have millimole/kilogram and around 10 micromole/kilogram sensitivity respectively [1].

All of the key mediators and cells in chronic inflammation represent potential targets for visualization and treatment of inflammatory disorders, and some of the inflammation biomarkers can already be investigated by PET [11]. Selection of highly specific probes for clinical imaging is a difficult task, since most of compounds with very promising in vitro and animal model results appear to be much less useful when applied in clinical settings, due to the differences in physiology and pathophysiology [12]. The existing probes for PET imaging could be applied to image a number of inflammation biomarkers, like [6]:

a. Metabolic activity of inflammatory cells (elevated metabolism of glucose and choline investigated using ${ }^{18} \mathrm{~F}-\mathrm{FDG}$ and ${ }^{18} \mathrm{~F}$-choline); b. Membrane markers of inflammatory cells, like translocator protein (TSPO), somatostatin receptor (SSTR), type 2 cannabinoid receptor $\left(\mathrm{CB}_{2} \mathrm{R}\right)$, formyl peptide receptor (FPR);

c. Inflammatory cytokines, like cyclooxygenase (COX), matrix metalloproteinases (MMPs), interleukin-2 (IL-2), tumor necrosis factor-a (TNF-a);

d. Targets on inflammation related vessels, like integrin receptor, vascular adhesion protein (VAP-1), vascular cell adhesion molecule (VCAM-1).

Most of the listed target cytokines or receptors are common both for inflammation and cancer and only a few could be considered inflammation-specific. Differential between benign and malignant processes might be possible when considering kinetics of FDG uptake [13], but still there are limitations and more research should be done in this direction. Cell labeling techniques, focused on monocytes or macrophages, using either radiotracers or paramagnetic NPs could be done to observe and quantify cell-trafficking, and could provide additional data in the sites of chronic inflammation, including peritumoral inflammation, with respect to prognosis and therapy response [14]. Again this technique is currently more experimental and needs more research to get a proper clinical translation. The other missing chain is related to techniques which would allow imaging the markers of transition from chronic inflammation to cancer, including imaging of damage-associated molecular patterns and cell atypia.

\section{Conclusion}

Inflammation is associated with development and malignant progression of most cancers [4]. Given the fact that an increasing number of research works address signal transduction pathways and molecular interaction networks in pathological states, a new growing number of targets and probes for chronic inflammation and cancer-associated imaging is becoming available $[15,16]$. Along with a growing demand for new imaging biomarkers for more effective comprehensive cancer care, there is a need to revise cancer screening paradigm in clinical imaging and shift it from identification of macroscopic lesions to recognition of precancer states. Current hybrid imaging techniques already provide some insight into functional molecular processes in chronic inflammation and cancer microenvironments; nevertheless they still face significant limitations both in terms of imaging approach specificity and integrity as well as in terms of clinical translation and approval of certain imaging techniques. More research is needed to bridge the gaps in imaging the transition from chronic inflammation to cancer, imaging cancer microenvironment and the transition from non-invasive to invasive cancer. In case of success, the results could be utilized for improving early cancer detection and cancer stage evaluation, therapy planning and response assessment, as well as for changing the prognostic indicators in oncology. 


\section{Cancer Therapy \& Oncology International Journal}

\section{Reference}

1. L Fass (2008) Imaging and cancer: A review. Molecular Oncology 2(2): 115-152.

2. Oishi Y, Manabe I (2016) Macrophages in age-related chronic inflammatory diseases. npj Aging and Mechanisms of Disease 2.

3. Szebeni GJ, Vizler C, Kitajka K, Puskas LG (2017) Inflammation and Cancer: Extra- and Intracellular Determinants of Tumor-Associated Macrophages as Tumor Promoters. Mediators of Inflammation.

4. Todoric J, Antonucci L, Karin M (2016) Targeting Inflammation in Cancer Prevention and Therapy. Cancer Prev Res (Phila) 9(12): 895905.

5. Nielsen SR, Schmid MC (2017) Macrophages as Key Drivers of Cancer Progression and Metastasis. Mediators of Inflammation 2017: 9624760

6. Wu C, Li F, Niu G, Chen X (2013) PET Imaging of Inflammation Biomarkers. Theranostics 3(7): 448-466.

7. Fujiwara N, Kobayashi K (2005) Macrophages in inflammation. Curr Drug Targets Inflamm Allergy 4(3): 281-286.

8. Haitian L, Weiming O, Chuanshu H (2006) Inflammation, a Key Event in Cancer Development. Mol Cancer Res 4(4): 221-233.

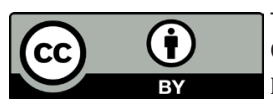

This work is licensed under Creative Commons Attribution 4.0 License

DOI: $10.19080 /$ CTOIJ.2017.04.555627
9. M Cutolo SP0127 (2015) Transition from Chronic Inflammation to Cancer: Immune Endocrine Synergies. Annals of the Rheumatic Diseases 74: 31-32.

10. Villanueva A, Luedde $\mathrm{T}$ (2016) The transition from inflammation to cancer in the liver. Clinical Liver Disease 8: 89-93.

11. Barreiro E, Bustamante V, Curull V, Gea J, López-Campos JL, et al (2016) Relationships between chronic obstructive pulmonary disease and lung cancer: biological insights. Journal of Thoracic Disease. 8(10): E1122-E1135.

12. Signore A, D’alessandria C, Annovazzi A, Scopinaro F (2002) Radiolabelled Cytokines for Imaging Chronic Inflammation 45.

13. van Waarde A, Elsinga PH (2008) Proliferation markers for the differential diagnosis of tumor and inflammation. Curr Pharm Des 14(31): 3326-339.

14. Van Hemert FJ, Voermans C, Van Eck-Smit BL, Bennink RJ (2009) Labeling monocytes for imaging chronic inflammation. Q J Nucl Med Mol Imaging 53(1): 78-88.

15. Higgins LJ, Pomper MG (2011) The Evolution of Imaging in Cancer: Current State and Future Challenges. Semin Oncology 38(1): 3-15.

16. Todoric J, Antonucci J, Karin M (2016) Targeting Inflammation in Cancer Prevention and Therapy. Cancer Prev Res (Phila) 9(12): 895905.

\section{Your next submission with Juniper Publishers will reach you the below assets}

- Quality Editorial service

- Swift Peer Review

- Reprints availability

- E-prints Service

- Manuscript Podcast for convenient understanding

- Global attainment for your research

- Manuscript accessibility in different formats

( Pdf, E-pub, Full Text, Audio)

- Unceasing customer service

Track the below URL for one-step submission https://juniperpublishers.com/online-submission.php 